\title{
Research of new 3D resistivity prospecting system electrical measuring device
}

\author{
${ }^{1}$ Haning $\mathrm{Xu}^{*}$, ${ }^{1} \mathrm{Hui} \mathrm{Xiao},{ }^{1}$ Zhenggen $\mathrm{Li},{ }^{1}$ Zhengjun Zeng, ${ }^{1}$ Juzhi Deng, \\ ${ }^{1}$ East China Institute of Technology
}

\begin{abstract}
In the exploration area, traditional high-density electrical prospecting was designed for 2D resistivity acquisition, the accuracy and sampling rate was low. A new type 3D resistivity acquisition system was designed in this thesis. Apparent resistivity data can be acquired by the new substation System , "E-SCAN" observation pattern was introduced for scanning poles, transition of A,M can be completed with distributed intelligent electrodes. The results of test shows that data are reliable, accurate and measuring cycle has been shortened considerably ,and the utility requirements of 3D resistivity prospecting can be achieved .
\end{abstract}

Keywords: three - dimensional resistivity ; E-SCAN; intelligent electrode ;Apparent resistivity

\subsection{Introduction}

At this stage, the advanced electrical prospecting instruments are all twodimensional high density resistivity instrument, such as : WDJD -2 and Superstring-R8. The method of two-dimensional data collection was conducted in a sequential manner and inefficient ${ }^{1}$. Because of the geological targets are three- dimensional structure, inversion interpretation with one or two dimensional inversion will inevitably produce err ${ }^{2}$. At present, only a few countries carried out a three dimensional Acquisition System and three - dimensional inversion of resistivity imaging studies such as the United States, France and Sweden ${ }^{3,4}$. Study of high accuracy three-dimensional exploration technology and greater depth measurements has become an important research direction.

A new high precision three-dimensional parallel DC electrical prospecting system was designed in this article : Measurement method was "E-SCAN " electrode scanning $^{5 \sim 8}$; Switched between measurement "M" and power supply "A" by smart electrodes, they could meet the requirements of single point of power supply and more parallel electric measurement ; Designing in three-dimensional Distributed

\footnotetext{
Haning Xu ( $\square)$

East China Institute of Technology, Department of Nuclear Engineering and Physical

Geography, 330013 Nanchang, China

e-mail:doud-ou@163.com
} 
structure , 24- bit high-precision A/D and ARM7 processor was the core of hardware circuit part . The host controlled multi-station to achieve synchronous acquisition of three- dimensional resistivity ${ }^{9,10}$. The three-dimensional inversion used RES2DINV software .

\section{Basic concepts of Apparent Resistivity}

DC resistivity method is based on conductivity of rocks and minerals in the earth's crust difference, prospecting and geological issues branch through observation and study of artificial distribution of current field . General supplied power by grounding electrode through the surface to underground, measured two points potential difference , represented powered electrode by "A , B", indicates the measurement electrodes by "M , N" . To the underground for $+\mathrm{I}$ in point "A", in point "B" into the ground for -I current, the "M, $\mathrm{N}$ "potential difference is :

$$
\Delta U_{M N}^{A}=\frac{\rho I}{2 \pi A M}-\frac{\rho I}{2 \pi A N}, \quad \Delta U_{M N}^{B}=-\frac{\rho I}{2 \pi B M}+\frac{\rho I}{2 \pi A N}
$$

The earth resistivity formula :

$$
\rho_{M N}^{A B}=K_{M N}^{A B} \frac{U_{M N}^{A B}}{I} \quad K_{M N}^{A B}=\frac{2 \pi}{\frac{1}{A M}-\frac{1}{A N}-\frac{1}{B M}+\frac{1}{B N}}
$$

"K" only related to the spatial location of electrode system, known as the coefficient of equipment.

Our study target is not uniform in real life, assumed it is uniform, calculated the resistivity based on the formula called the apparent resistivity. It is an underground media response to resistance. Any of the Four-pole arrangements shown in figure 1.1 .

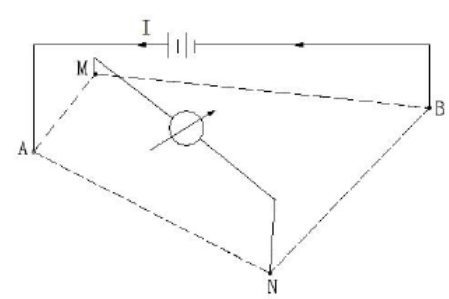

Fig 2.1 Any of the Four-pole arrangements

\section{Observation and working devices}

\section{1 "E-SCAN"}

Geophysicists Li. Oldenburg and Shore proposed " E-SCAN " measurement method for three dimensional resistivity measurement in 1992. The 1st electrode 
supplied power, all the other electrodes measured to achieve $\mathrm{N}-1$ data . The 2nd electrode supplied power ${ }^{11}$, other measured electrode (According to the principle of exchange : 1st electrodes supply power and 2nd measurements are equivalent with 2nd electrodes supply power and 1st measurements ). And so on , got the $\mathrm{N}-2$ Data, total number of measurements is $\mathrm{N} \times(\mathrm{N}-1) / 2$ 。

"Cross-diagonal" method could also be used to only measure parallel to the current electrode direction, vertical or 450 high number of electrode potential value ( fig3.1 ). In this way, the workload of measurement could be reduced, it was easy to convert pole-dipole, dipole-pole and dipole- dipole data 。

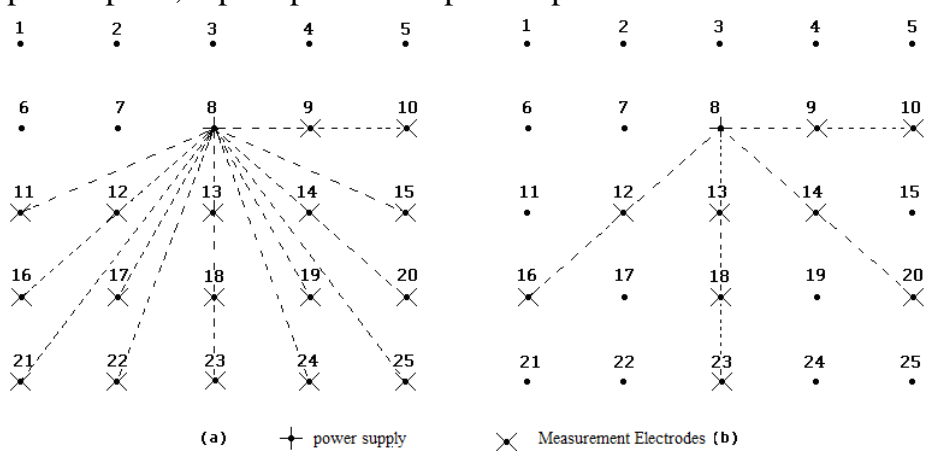

Fig3.1 "E-SCAN" Measurement diagram

The system adopts the multi-channel observed at the same time : After 256 electrode arrangement , electrode conversion used program control in "E-SCAN" to measure not only the high number of electrodes but also electrode for Measurement of Low . In this way the subsequent three-dimensional inversion and visualization was formatted.

\subsection{Pole-pole}

When " B " and " N" are in infinity:

$$
\rho_{s}^{A M}=K^{A M} \frac{U_{M}}{I_{A B}}, \quad K^{A M}=2 \pi A M
$$

Two-pole device sensitivity of contour is neither horizontal nor vertical (fig3.2) .It has moderate horizontal and vertical resolution, while probing depth is large, observed signal is weaker than " Wenner " and " Wenner -Schlumberger ", higher than the Dipole - Dipole system . It is commonly used in three - dimensional method. 


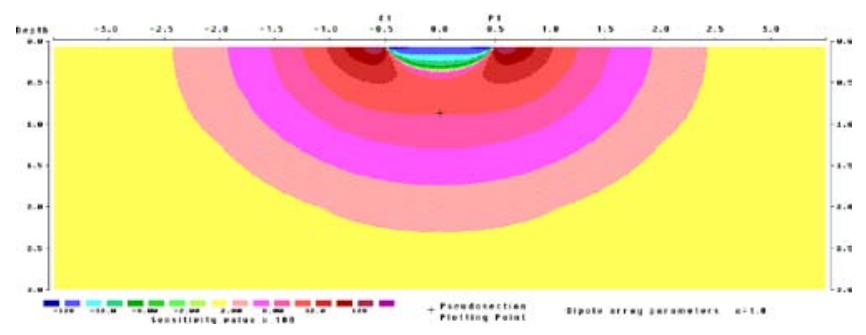

Fig3.2 Sensitivity of pole-pole

\section{Hardware design}

High precision 24-bit ADC chip ADS1258 was used in this article . it has fixed $125 \mathrm{ksps}$ data rate of the channel . Sampling error is less than $0.0003 \%$ with $\pm 2.5 \mathrm{~V}$ voltage acquisition. Using High-speed 32-bit ARM7TDMI LPC2378 processor was used as the main control Chip, it's frequency was $72 \mathrm{MHz}$. For improving the anti - interference performance of the system , electromagnetic compatibility , signal integrity and power integrity was used in 4-layer PCB board design .

Two main parts of circuit design : the analog signal conditioning circuit before the AD conversion and the digital signal processing circuits .

Analog signal conditioning circuit module as shown in figure 4.1 . The 16channel electrodes signal was inputted multi-channel analog switch though $\pm 12 \mathrm{~V}$ overvoltage protection . With low offset voltage and low on-resistance integrated chip ADG526, one channel could be selected from 16 channels . Band-stop module was designed to filter 48 52HZ interference, it could effectively improve the collection of anti-interference performance in the field environment . Considering the input signal low frequency and small amplitude characteristics . The signal was amplified before low pass filter ( $80 \mathrm{HZ}$ ), using programmable gain amplifier PGA205, magnification program is set to 2, 4 and 8 . Signal amplifier selection of second order with PGA205, the magnification can be set to 10, 100 and 1000.After the adoption of two-order amplifier, the input signal adjustment in the context of ADS1258 the best conversions.

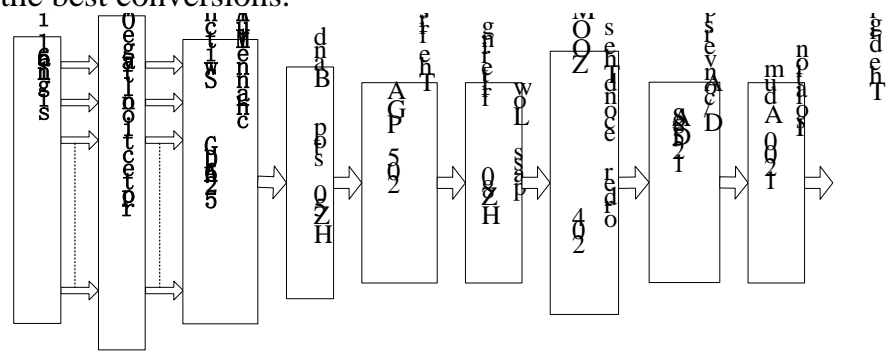

Fig4.1 Signal conditioning circuit module 
Bipolar voltage of $\pm 2.5 \mathrm{~V}$ differential-mode sampling methods was designed in ADS1258, it could effectively avoided benchmark error . Acquisition mode was set fixed channel mode to avoid channel interference errors . With SPI bus communication between the ADS1258 and ARM7, realization of data exchange between the microcontroller and AD. The ADS1258 crossover analog circuits and digital circuits, in order to reduce signal interference, used optical coupling isolation analog switch ADuM1200ARZ control signals .

Microprocessor LPC2378 was the core of digital circuit module, included reset control circuit, CAN bus interface, RS485 communication interface . The station and PC communicated with CAN bus, the RS485 interface implementation substation communicates with the smart electrode . Collection process showed in figure4.2.

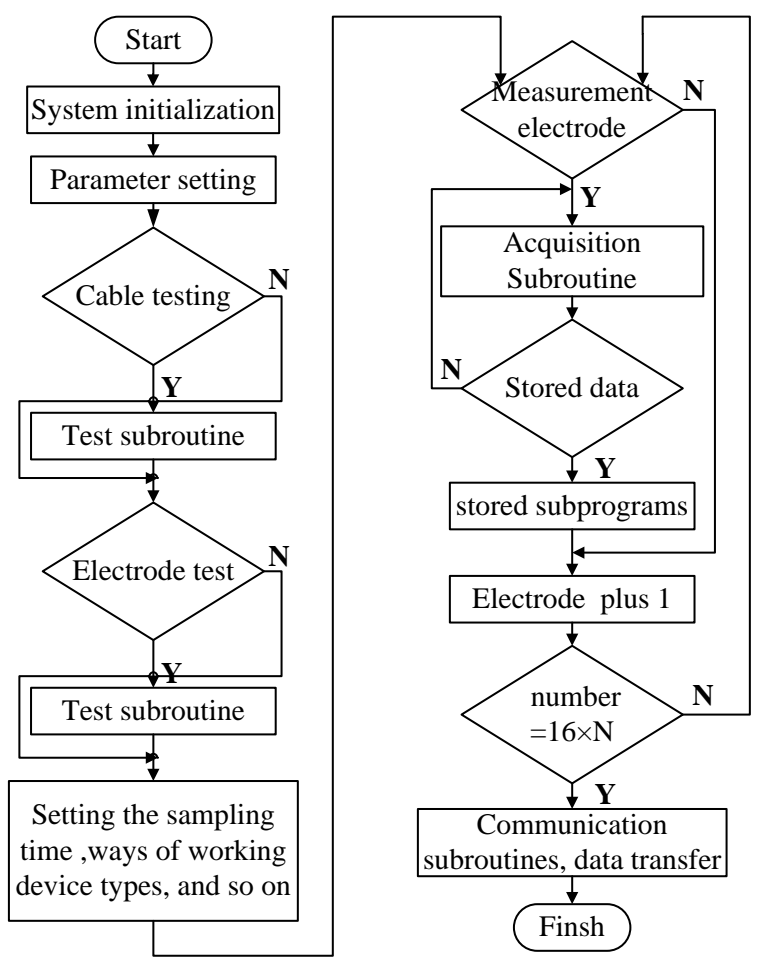

Fig4.2 Collection System Software flowchart 


\section{Validation Tests}

\subsection{Test conditions and procedures}

Test conditions: East China institute of technology three-dimensional electromagnetic model experiment tank . Side length is $6 \mathrm{~m}$, Water depth is $3 \mathrm{~m}$. 16×16 measurement of three -dimensional grid was put in the pool, the adjacent electrodes margin $a=10 \mathrm{~cm}$. High impedance Board was in the central of $\operatorname{grid}(600 \mathrm{~mm} \times$ $400 \mathrm{~mm}$ ), Top of the plate depth was $10 \mathrm{~cm}$. The determinant electrode was numbered , such as $1-1,1-2 、 \ldots \ldots \ldots . .16-15,16-16.1-1$ is 1 st electrode in 1 st row , 16-16 is 16th electrode in 16th row . The specific method, as follows:

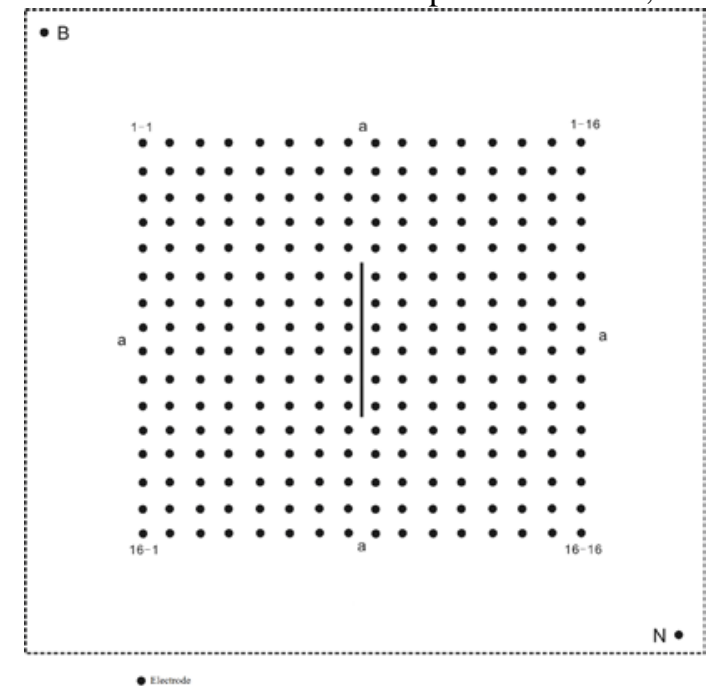

Fig 5.1 Electrode layout

1. Observation by three-dimensional E-Scan ( Pole-Pole array ).

2. Full Channels measurement。The 1st electrode power supply, the 2nd to 256th electrode measured data at the same time . And so on , the 2nd electrode power supply, all the other electrodes measured data .

3. 65,536 data was measured at a time , According to the principle of potential swap, processing the same average depth point data , the actual data points is 32,768.

4.Finally, the data into pole-pole device of three-dimensional electron inversion data formats. 


\subsection{Analysis of test results}

Horizontal slice level of 7th showed from light to deep resistivity slices . Figure 5.2(a) showed the 3rd vertical in X direction from top to bottom slicing . The 3rd slice through the plate can be seen from figure 1 to 3, apparent glaringly, and the high resistance of contour round closed conformity with the position of the board . Figure 5.2(c) showed the 3rd vertical slice in Y direction ( $\mathrm{y}-\mathrm{z})$.

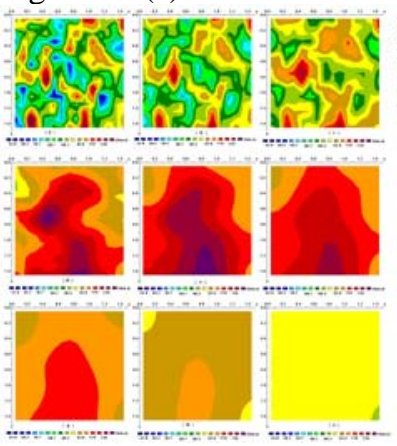

(a)

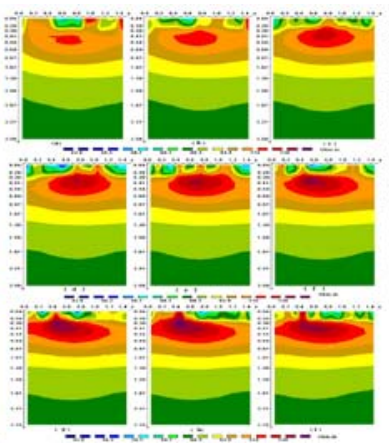

(b)

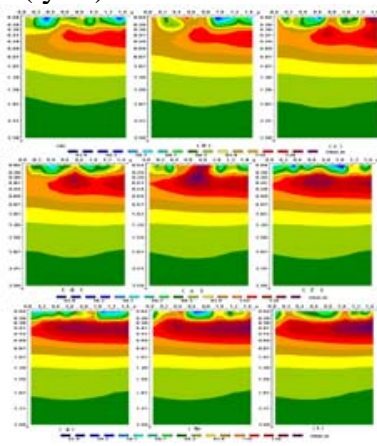

(c)

Fig5.2 (a) Horizontal slice from 1st to 3rd layer (b)slice from 4th to 6th layer in X direction (c) slice from 4th to 6th layer in $\mathrm{Y}$ direction

\section{Conclusions}

Three-dimensional resistivity acquisition system can be widely applied to water, workers, the central geological prospecting and exploration of mineral resources . The new three-dimensional resistivity of collection systems overcome the current General Electric Prospecting data acquisition system for measuring accuracy low and slow , the main disadvantage of the poor real-time performance, filled the blank of three - dimensional electrical prospecting in China, promoted the country's resources investigation . It had a positive effect geological exploration to the development of high resolution three - dimensional .

\section{Acknowledgments:}

The research described in this paper was funded by Projects of International Cooperation (2007DFA20790), Science \& technology Project of Jiangxi Province Education Department (Jiangxi Province Education Finance [2011]243) , Jiangxi Province Science Foundation for Youths (20122BAB211016) .

\section{References}

1. Petrick W R Jr, Sill W R, Ward S H(1981) . Three-dimensional resistiity inversion using alphacenters. Geophysics , 46(8):1148-1162. 
2. Sasaki Y(1994).3-D resistivity inversion using the finite-element method.Geophysics, 59:18391850.

3. HORE G(1992). E-SCAN resource mapping: multi directional electrical surveys[M ]. Premier Geophysics Inc. , Richmond, B. C. , Canada..428-440.

4. LOKEM H, BARKER R D( 1996). Practical techniques for 3D resistivty survey s and data inversion [ J ]. Geophysical Prospecting, 44: 499-523.

5.LOKEM H, BARKER R D(1994). Least-squares deconvolution of apparent resistivity pseudosections [ J]. Geophysics,160: 1682-1690.

6. Osher S, Sethian JA (1988) Fronts propagating with curvature-dependent speed: algorithms based on Hamilton- Jacobi formulations, J. Com Phys, 79 (1):12-49.

7. Chan T, Vese L (2001) Active contours without edges, IEEE Trans on Image Proc,10:266-277.

8. SH IMA H(1990). Two-dimensional automatic resistivity inversion technique using alpha centers [ J]. Geophysics, 55: 682-694.

9. Vese L, Chan T (2002) A multiphase level set framework for image segmentation using the Mum ford and Shah model, Int J Comp Vis, 50:271-293.

10.Zhu SC, Yuille A (1996) Region competition: Unifying snakes, region growing, and Bayes/MDL for multi band image segmentation, IEEE Trans Pattern Anal Mach Intell.18(9):884-900.

11.Tang Hongzhi ,Liu Qingcheng , Yin Zhiyong , Bao Xun(2011).Experimental research of LQC-I 3D resistivity survey system. Chinese Journal of Scientific Instrument.32(5):1058-1061. 